\title{
EFFECT OF TOCILIZUMAB IN MORTALITY AMONG PATIENTS WITH SEVERE AND CRITICAL COVID-19: EXPERIENCE IN A THIRD-LEVEL MEDICAL CENTER
}

\author{
Bernardo A. Martínez-Guerra ${ }^{1}$, Nereyda A. de-León-Cividanes ${ }^{1}$, Karla M. Tamez-Torres², \\ Carla M. Román-Montes ${ }^{1}$, Sandra Rajme-López ${ }^{1}$, Edgar Ortiz-Brizuela ${ }^{1}$, Carlos A. \\ Aguilar-Salinas ${ }^{3}$, Juan Sierra-Madero ${ }^{1}$, José Sifuentes-Osornio ${ }^{4}$, Alfredo Ponce-de-León ${ }^{1}$, \\ AND MARÍA F. GONZÁLEZ-LARA ${ }^{2 *}$ \\ ${ }^{1}$ Department of Infectious Diseases, ${ }^{2}$ Clinical Microbiology Laboratory, ${ }^{3}$ Department of Nutrition, and \\ ${ }^{4}$ Department of Medicine. Instituto Nacional de Ciencias Médicas y Nutrición Salvador Zubirán, Mexico City, Mexico
}

\begin{abstract}
Background: Trials evaluating safety and efficacy of tocilizumab in coronavirus disease 19 (COVID-19) show contradictory results. Objective: The objective of the study was to evaluate the effect of tocilizumab in hospital mortality among patients with severe COVID-19 in a third-level medical center. Methods: This prospective cohort study included patients with severe and critical COVID-19. Primary outcome was death during hospitalization. Secondary outcomes included invasive mechanical ventilation (IMV), days on IMV, ventilator-free days (VFDs), length of hospital stay (LOS), and development of hospitalacquired infections (HAls). Bivariate, multivariate, and propensity score matching analysis were performed. Results: During the study period, $99 / 794$ (12\%) patients received tocilizumab. Male patients, health care workers, and patients with increased inflammatory markers received tocilizumab more frequently. No difference in hospital mortality was observed between groups ( $34 \%$ vs. $34 \%, p=0.98$ ). Tocilizumab was not independently associated with mortality. No significant treatment effects were observed in propensity score analysis. IMV was more frequent ( $46 \%$ vs. $11 \%, p<0.01$ ) and LOS was longer (12 vs. 7 days, $p<0.01$ ) in the tocilizumab group, reflecting increased severity. Although HAls were more frequent in the tocilizumab group ( $22 \%$ vs. $10 \%, p<0.01$ ), no difference was seen after adjusting for IMV $(38 \%$ vs. $40 \%, p=0.86$ ). Conclusions: In our study, tocilizumab was not associated with decreased hospital mortality among patients with severe COVID-19. (REV INVEST CLIN. 2022;74(1):40-50)
\end{abstract}

Keywords: Tocilizumab. COVID-19. SARS-CoV-2. Mexico.

*Corresponding author:

María F. González-Lara

E-mail: fergonla@gmail.com
Received for publication: 30-07-2021

Approved for publication: 08-09-2021

DOI: $10.24875 /$ RIC.21000404

0034-8376 / (c) 2021 Revista de Investigación Clínica. Published by Permanyer. This is an open access article under the CC BY-NC-ND license (http://creativecommons.org/licenses/by-nc-nd/4.0/). 


\section{INTRODUCTION}

The lack of effective treatment options to reduce COVID-19 associated mortality, disease progression, and the need for invasive mechanical ventilation (IMV) remains a problem. Severe forms of COVID-19 are associated with an increased systemic inflammation and elevation of markers such as $\mathrm{C}$-reactive protein $(C R P)$, lactate dehydrogenase (LDH), D-Dimer, ferritin, interleukin (IL)-6, IL-8, and TNF- $\alpha^{1,2}$. Since the first descriptions of COVID-19, anti-inflammatory therapies have been explored. The RECOVERY trial demonstrated a mortality reduction with dexamethasone in hypoxic patients ${ }^{3}$. Numerous anti-inflammatory therapies and immunomodulating agents have been proposed to treat severe forms of COVID-194.

Tocilizumab is a recombinant anti-IL-6 monoclonal antibody that binds to membrane and soluble IL- 6 receptors ${ }^{5}$, currently recommended in the treatment of rheumatoid arthritis and cytokine release syndrome $^{6,7}$. Its use for COVID-19 was first described in small case series where clinical and radiographic improvements were observed after the administration of the drug 8,9 . While numerous cohorts have reported successful outcomes, such results have not been consistently observed in randomized control trials (RCTs) and meta-analysis ${ }^{10-19}$. Updated guidelines consider the use of tocilizumab among patients with progressive or critical COVID-19 in addition to corticosteroids ${ }^{20-23}$. Outcomes of tocilizumab administration must be further explored. Therefore, we conducted a cohort study to evaluate the effect of tocilizumab on hospital mortality among patients with severe or critical COVID-19.

\section{METHODS}

\section{Patients and setting}

This prospective cohort study was conducted in a tertiary care center in Mexico City that was converted into a COVID-19 dedicated facility since March 16, 2020. Reorganization included expansion of the intensive care unit (ICU) and redistribution of nursing and medical staff. Data from all consecutive patients admitted with severe and critical COVID-19 between March 20 and June 10 were registered. Patients were followed up from admission to death or discharge. A severe case was defined by the presence of any of the following: respiratory rate $\geq 30$ breaths per minute, $\mathrm{SpO}_{2}<93 \%, \mathrm{PaO}_{2} / \mathrm{FiO}_{2}$ ratio $<300$, or $\geq 50 \%$ lung involvement by chest computed tomography (CT). A critical case was considered when IMV, shock, or multiorgan failure were present ${ }^{24}$. Demographic, clinical, laboratory, imaging, and outcome data were obtained from the electronic medical record. Among patients who received tocilizumab, laboratory test results from $24 \mathrm{~h}$ before and $48 \mathrm{~h}$ after the first dose of tocilizumab were registered. Previously validated severity and prognostic scores (MLS-COVID-1925 and Nutri$\mathrm{CoV}$ score $^{26}$ ) were registered retrospectively. Primary outcome was death during hospitalization. Secondary outcomes included IMV, days on IMV, ventilator-free days (VFDs) at 28 days, length of stay (LOS), and development of a culture-proven hospital-acquired infection (HAI). VFDs was defined as the number of days being alive and free of IMV for $24 \mathrm{~h}$; patients discharged from the hospital before 28 days were considered alive and free of IMV at day 28. Patients with moderate disease or a LOS $<24 \mathrm{~h}$ were excluded from the study. The study was approved by the Institutional Review Board (Ref. number 3333). Written informed consent was waived because of the observational nature of the study.

\section{Laboratory procedures}

SARS-CoV-2 testing was performed on nasopharyngeal swab samples. NucliSens easyMAG system (bioMérieux, Boxtel, the Netherlands) was used for nucleic acid extraction. Real-time reverse transcription-polymerase chain reaction was processed on Applied Biosystems 7500 thermocycler (Foster City, CA, USA) using primers and conditions described elsewhere ${ }^{27}$.

\section{Tocilizumab administration}

Intravenous administration of tocilizumab was prescribed by the treating physicians after evaluation of each case, considering possible contraindications (e.g., documented infection other than COVID-19 and known hypersensitivity to tocilizumab). All patients signed an informed consent addressing the potential risks and benefits of tocilizumab therapy known at the time. Because tocilizumab was not widely available in our center, dosing was variable according to 
Figure 1 . Number of subjects included in the study and follow-up.

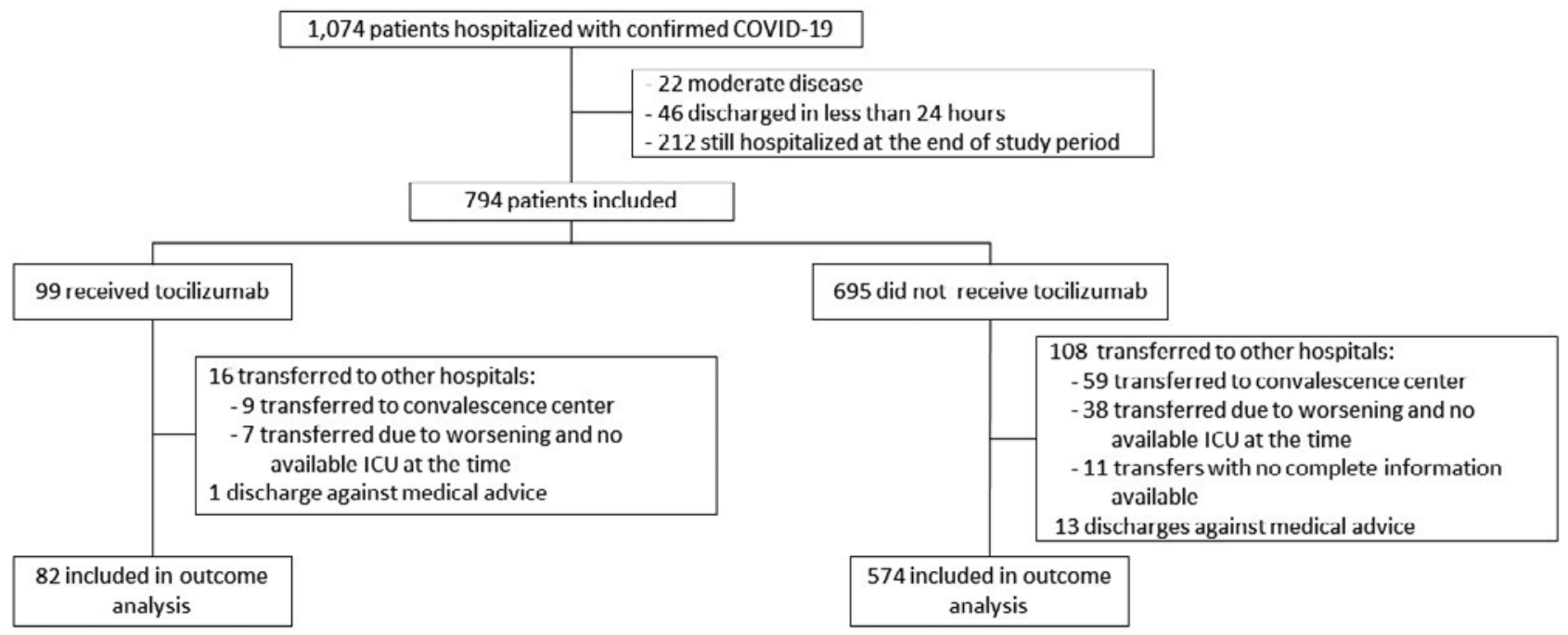

local availability and individual patient's resources. All patients received the standard of care. Of note, dexamethasone was not a standard of care in this center during the study period.

\section{Statistical analysis}

Descriptive analysis was made through mean, standard deviation, median, and interquartile range (IQR), as appropriate. Comparative analysis was made using $\chi^{2}$, Fisher's exact test, $t$-test for independent samples, two-sample rank sum tests, and sign-rank tests. Relative risk (RR) for hospital mortality and 95\% confidence interval $(95 \% \mathrm{Cl})$ were calculated for each variable in the bivariate analysis. Multivariate analysis using multiple regression was made; variables with $p$ $<0.05$ in bivariate analysis and those with biological plausibility (e.g., administration of tocilizumab) were included. To estimate treatment effects and minimize selection bias, a propensity score analysis using a matching method was performed. To estimate the propensity score, the treatment received was regressed in a logistic regression model. Baseline variables that could affect the outcome and influence the treatment selection were included in the model. Patients were matched on the logit of the propensity score using calipers of width of 0.1 or less of the standard deviation of the estimated propensity score and on a 1:1 ratio using control replacements if needed. To adequately specify the model, a comparison between variances of continuous variables and standardized means in the matched sample was made. A regression analysis within the matched sample was performed. Finally, the average treatment effect was estimated. A two-tailed $p<0.05$ was considered statistically significant. The analyses were performed using STATA version 15.1 (Texas, USA).

\section{RESULTS}

A total of 794 patients with severe or critical COVID-19 were included, of whom 99 (12\%) received tocilizumab (Fig. 1). Median age was 52 (IQR 43-62) years and 489 (62\%) were male. The median body mass index (BMI) was 29.7 (IQR 26.7-33.2) kg/m², $364(46 \%)$ had a BMI > $30 \mathrm{~kg} / \mathrm{m}^{2}, 216$ (27\%) had type 2 diabetes mellitus (DM), 253 (32\%) hypertension, and 45 (6\%) were immunocompromised. The most common cause of immunosuppression was pharmacological in 25/45 (56\%). Very high-risk category on MLS-COVID-19 and Nutri-CoV scores was observed in 166 (21\%) and 366/768 (48\%), respectively, and no differences between groups were observed. Patients on tocilizumab were more frequently male (74 [74\%] vs. 416 [60\%], p < 0.01), health care workers (12 [12\%] vs. 31 [5\%], p < 0.01), and less likely to have hypertension (19 [19\%] vs. 234 [34\%] $\mathrm{p}<0.01)$. No other differences were observed between groups (Table 1 ). 
Table 1. Baseline demographic and treatment characteristics

\begin{tabular}{|c|c|c|c|c|}
\hline Characteristic & $\begin{array}{l}\text { All patients, } \\
(n=794) \\
(100 \%)\end{array}$ & $\begin{array}{l}\text { Received tocilizumab, } \\
\qquad \begin{array}{c}(\mathrm{n}=99) \\
(12 \%)\end{array}\end{array}$ & $\begin{array}{l}\text { Did not receive } \\
\text { tocilizumab, } \\
(n=695) \\
(88 \%)\end{array}$ & $\mathrm{p}$ \\
\hline $\begin{array}{l}\text { Male sex, } \\
\mathrm{n}(\%)\end{array}$ & $\begin{array}{c}489 \\
(61.6)\end{array}$ & $\begin{array}{c}73 \\
(73.7)\end{array}$ & $\begin{array}{c}416 \\
(59.9)\end{array}$ & 0.008 \\
\hline Age, years, median (IQR) & $\begin{array}{c}52 \\
(43-62)\end{array}$ & $\begin{array}{c}51 \\
(43-61)\end{array}$ & $\begin{array}{c}52 \\
(43-62)\end{array}$ & 0.54 \\
\hline $\begin{array}{l}\text { Body mass index, } \mathrm{kg} / \mathrm{m}^{2} \text {, } \\
\text { median (IQR) } \\
\mathrm{n}=755\end{array}$ & $\begin{array}{c}29.7 \\
(26.7-33.2)\end{array}$ & $\begin{array}{c}26.7 \\
(27.0-33.9)\end{array}$ & $\begin{array}{c}29.7 \\
(26.7-33.2)\end{array}$ & 0.7059 \\
\hline $\begin{array}{l}\text { Obesity, } \\
\text { n (\%) }\end{array}$ & $\begin{array}{c}364 \\
(46.1)\end{array}$ & $\begin{array}{c}48 \\
(48.5)\end{array}$ & $\begin{array}{c}316 \\
(45.7)\end{array}$ & 0.607 \\
\hline $\begin{array}{l}\text { Diabetes mellitus, } \\
\mathrm{n}(\%)\end{array}$ & $\begin{array}{c}216 \\
(27.2)\end{array}$ & $\begin{array}{c}25 \\
(25.3)\end{array}$ & $\begin{array}{c}191 \\
(27.5)\end{array}$ & 0.641 \\
\hline $\begin{array}{l}\text { Arterial hypertension, } \\
\mathrm{n}(\%)\end{array}$ & $\begin{array}{c}253 \\
(31.9)\end{array}$ & $\begin{array}{c}19 \\
(19.2)\end{array}$ & $\begin{array}{c}234 \\
(33.7)\end{array}$ & 0.004 \\
\hline $\begin{array}{l}\text { Chronic obstructive pulmonary } \\
\text { disease, } \mathrm{n}(\%)\end{array}$ & $\stackrel{9}{(1.1)}$ & $\begin{array}{l}2 / 99 \\
(2.0)\end{array}$ & $\begin{array}{l}7 / 695 \\
(1.0)\end{array}$ & 0.312 \\
\hline $\begin{array}{l}\text { Asthma, n (\%) } \\
\mathrm{n}=793\end{array}$ & $\begin{array}{c}9 \\
(1.1)\end{array}$ & $\begin{array}{l}2 / 98 \\
(2.0)\end{array}$ & $\begin{array}{c}7 / 695 \\
(1.1)\end{array}$ & 0.308 \\
\hline $\begin{array}{l}\text { Cardiovascular disease, } \mathrm{n}(\%) \\
\mathrm{n}=793\end{array}$ & $\begin{array}{c}36 \\
(4.5)\end{array}$ & $\begin{array}{l}6 / 98 \\
(6.1)\end{array}$ & $\begin{array}{l}30 / 695 \\
(4.3)\end{array}$ & 0.43 \\
\hline $\begin{array}{l}\text { Immunosuppression, } \\
\mathrm{n}(\%)\end{array}$ & $\begin{array}{c}45 \\
(5.7)\end{array}$ & $\begin{array}{c}2 \\
(2.0)\end{array}$ & $\begin{array}{c}43 \\
(6.2)\end{array}$ & 0.106 \\
\hline $\begin{array}{l}\text { Chronic kidney disease, } \\
\mathrm{n}(\%)\end{array}$ & $\begin{array}{c}24 \\
(3.0)\end{array}$ & 0 & $\begin{array}{c}24 \\
(3.5)\end{array}$ & 0.06 \\
\hline $\begin{array}{l}\text { Current smokers, n (\%) } \\
n=785\end{array}$ & $\begin{array}{c}117 \\
(14.9)\end{array}$ & $\begin{array}{l}10 / 97 \\
(10.3)\end{array}$ & $\begin{array}{l}107 / 688 \\
(15.6)\end{array}$ & 0.175 \\
\hline $\begin{array}{l}\text { Healthcare worker, n (\%) } \\
n=792\end{array}$ & $\begin{array}{c}43 \\
(5.4)\end{array}$ & $\begin{array}{l}12 / 99 \\
(12.1)\end{array}$ & $\begin{array}{l}31 / 693 \\
(4.5)\end{array}$ & 0.007 \\
\hline $\begin{array}{l}\text { Charlson comorbidity index } \geq 2 \text {, } \\
\mathrm{n}(\%)\end{array}$ & $\begin{array}{c}328 \\
(41.3)\end{array}$ & $\begin{array}{c}36 \\
(36.4)\end{array}$ & $\begin{array}{c}292 \\
(42.0)\end{array}$ & 0.285 \\
\hline $\begin{array}{l}\mathrm{SpO}_{2} \leq 90 \%, \mathrm{n}(\%) \\
\mathrm{n}=770\end{array}$ & $\begin{array}{c}721 \\
(93.6)\end{array}$ & $\begin{array}{l}89 / 94 \\
(94.7)\end{array}$ & $\begin{array}{l}632 / 676 \\
(93.5)\end{array}$ & 0.823 \\
\hline \multicolumn{5}{|l|}{$\begin{array}{l}\text { Nutri-CoV category, n (\%) } \\
n=768\end{array}$} \\
\hline Low risk & $\begin{array}{c}28 \\
(3.7)\end{array}$ & $\begin{array}{l}2 / 94 \\
(2.1)\end{array}$ & $\begin{array}{c}26 / 674 \\
(3.9)\end{array}$ & 0.114 \\
\hline Moderate risk & $\begin{array}{c}131 \\
(17.1)\end{array}$ & $\begin{array}{l}12 / 94 \\
(12.8)\end{array}$ & $\begin{array}{l}119 / 674 \\
(17.7)\end{array}$ & \\
\hline High risk & $\begin{array}{c}243 \\
(31.6)\end{array}$ & $\begin{array}{l}40 / 94 \\
(42.6)\end{array}$ & $\begin{array}{l}203 / 674 \\
(30.1)\end{array}$ & \\
\hline Very high risk & $\begin{array}{c}366 \\
(47.7)\end{array}$ & $\begin{array}{l}40 / 94 \\
(42.6)\end{array}$ & $\begin{array}{l}326 / 674 \\
(48.4)\end{array}$ & \\
\hline $\begin{array}{l}\text { Lymphopenia }<800 \text { cells } / \mu \mathrm{L}, \mathrm{n}(\%) \\
\mathrm{n}=788\end{array}$ & $\begin{array}{c}426 \\
(54.1)\end{array}$ & $\begin{array}{l}62 / 99 \\
(62.6)\end{array}$ & $\begin{array}{l}364 / 689 \\
(52.8)\end{array}$ & 0.067 \\
\hline $\begin{array}{l}\text { C-reactive protein >10 mg/dL, n (\%) } \\
n=766\end{array}$ & $\begin{array}{c}553 \\
(72.2)\end{array}$ & $\begin{array}{l}76 / 97 \\
(78.4)\end{array}$ & $\begin{array}{l}477 / 669 \\
(71.3)\end{array}$ & 0.148 \\
\hline $\begin{array}{l}\text { D-dimer >1000 ng/mL, n (\%) } \\
\mathrm{n}=762\end{array}$ & $\begin{array}{c}272 \\
(35.7)\end{array}$ & $\begin{array}{c}25 \\
(26.6)\end{array}$ & $\begin{array}{c}247 \\
(37.0)\end{array}$ & 0.049 \\
\hline
\end{tabular}


Table 1. Baseline demographic and treatment characteristics (continued)

\begin{tabular}{|c|c|c|c|c|}
\hline Characteristic & $\begin{array}{l}\text { All patients, } \\
(n=794) \\
(100 \%)\end{array}$ & $\begin{array}{c}\text { Received tocilizumab, } \\
\qquad \begin{array}{c}(n=99) \\
(12 \%)\end{array}\end{array}$ & $\begin{array}{l}\text { Did not receive } \\
\text { tocilizumab, } \\
(n=695) \\
(88 \%)\end{array}$ & $\mathrm{p}$ \\
\hline $\begin{array}{l}\text { Empiric antibiotic treatment, } \\
\mathrm{n}(\%)\end{array}$ & $\begin{array}{c}744 \\
(93.7)\end{array}$ & $\begin{array}{c}95 \\
(96)\end{array}$ & $\begin{array}{l}649 \\
(93.4)\end{array}$ & 0.505 \\
\hline $\begin{array}{l}\text { Treatment with hydroxychloroquine, } \\
\mathrm{n}(\%)\end{array}$ & $\begin{array}{c}219 \\
(27.6)\end{array}$ & $\begin{array}{c}33 \\
(33.3)\end{array}$ & $\begin{array}{c}186 \\
(26.8)\end{array}$ & 0.171 \\
\hline $\begin{array}{l}\text { Adjuvant steroids, } \\
\mathrm{n}(\%)\end{array}$ & $\begin{array}{c}73 \\
(9.2)\end{array}$ & $\begin{array}{c}9 \\
(9.1)\end{array}$ & $\begin{array}{c}64 \\
(9.2)\end{array}$ & 0.97 \\
\hline $\begin{array}{l}\text { ICU care on admission, } \\
\mathrm{n}(\%)\end{array}$ & $\begin{array}{c}112 \\
(14.1)\end{array}$ & $\begin{array}{c}17 \\
(17.2)\end{array}$ & $\begin{array}{c}95 \\
(13.7)\end{array}$ & 0.349 \\
\hline $\begin{array}{l}\text { Use of invasive ventilation } \\
\text { at admission, } \mathrm{n}(\%)\end{array}$ & $\begin{array}{c}103 \\
(13.0)\end{array}$ & $\begin{array}{c}17 \\
(17.2)\end{array}$ & $\begin{array}{c}86 \\
(12.4)\end{array}$ & 0.184 \\
\hline
\end{tabular}

ICU: intensive care unit; IQR: interquartile range; kg: kilogram; m: meter; mg/dL: milligram per deciliter; ng/mL: nanogram per deciliter; $\mu \mathrm{L}$ : microliter.

On admission, the median oxygen saturation was $83 \%$ (IQR 70-88), a NEWS score $\geq 7$ was found in 580 ( $87 \%)$, a MuLBSTA score $\geq 11$ in 156 (20\%), and 785 (99\%) had multiple lobe involvement in the initial chest CT scan. There were no differences among severity scores between groups. The median time from symptom onset to hospital admission was shorter in the tocilizumab group (7 [IQR 5-9] days vs. 8 [IQR 6-10] days, $p<0.05$ ) (Table S1, Supplementary material). Laboratory test results on admission did not differ between groups, except for D-dimer (Table S2). Median $\mathrm{PaO}_{2} / \mathrm{FiO}_{2}$ was 197 in 766 patients. Interferon-gamma release assay (IGRA) for latent tuberculosis was positive more frequently among patients who received tocilizumab (11/74 [15\%] vs. 9/138 [7\%], $\mathrm{p}<0.05$ ).

On admission, $103 / 794$ patients (13\%) were placed on IMV. Chloroquine/hydroxychloroquine and steroids were used in 219/794 (28\%) and 73/794 (9\%) patients, respectively. Among patients who received steroids, 40/73 (55\%) received hydrocortisone for refractory circulatory shock, $17 / 73(23 \%)$ received methylprednisolone, and 16/73 (22\%) received lowdose prednisone due to prior chronic steroid treatment. Empiric antibiotic treatment was prescribed in $744 / 794$ (94\%) patients, and $\beta$-lactams were the most commonly prescribed agents. Therapeutic anticoagulation during follow-up was more commonly used in the tocilizumab group (40/99 [40\%] vs. 66/695 [10\%], $p<0.01$ ).

\section{Hospital follow-up and tocilizumab administration}

At $72 \mathrm{~h}$ after hospital admission, the median lymphocyte count was 901 cells $/ \mu \mathrm{L}$ and the median $\mathrm{PaO}_{2} /$ $\mathrm{FiO}_{2}$ ratio was 115.4; no differences between groups were observed. Patients who received tocilizumab had higher median concentrations of CRP (17.8 vs. 11.3 $\mathrm{mg} / \mathrm{dL}, \mathrm{p}<0.01$ ), LDH (409 vs. $314 \mathrm{U} / \mathrm{L}, \mathrm{p}<0.01$ ), ferritin ( 983 vs. $659 \mathrm{ng} / \mathrm{mL}, \mathrm{p}<0.01$ ), and procalcitonin ( 0.52 vs. $0.26 \mathrm{ng} / \mathrm{mL}, \mathrm{p}<0.05$ ). No differences were seen in liver function tests, blood cell counts, D-dimer, creatine phosphokinase, and troponin I (Table S3).

The median time from symptom onset to tocilizumab administration was 11 (IQR 9-13) days and the median time from admission to tocilizumab 4 (IQR 2-6) days. Thirty patients (30\%) received the drug $24 \mathrm{~h}$ or more after IMV onset. The median total dose was 400 mg (range: 200-800 mg). Five patients received two doses of tocilizumab within the first $24 \mathrm{~h}$ after the first dose. After tocilizumab, the median CRP level decreased from 19.2 to $4.8 \mathrm{mg} / \mathrm{dL}(p<0.01)$, and median alanine aminotransferase (ALT) and aspartate aminotransferase (AST) levels increased from 40.3 to 58.5 and 47.7 to $70.6 \mathrm{U} / \mathrm{L}(\mathrm{p}<0.01)$, respectively. The median lymphocyte count increased from 756.8 to 916.8 cells $/ \mu \mathrm{L}(\mathrm{p}<0.05)$ while the median neutrophil count decreased from 6483 to 4415 cells $/ \mu \mathrm{L}$ $(p<0.01)$. No differences were seen in other 
Table 2. Severe and critical COVID-19 patients' outcomes regarding tocilizumab use

\begin{tabular}{|c|c|c|c|c|}
\hline Outcomes & $\begin{array}{l}\text { All patients, } \\
(n=656) \\
(100 \%)\end{array}$ & $\begin{array}{l}\text { Received tocilizumab, } \\
\qquad(\mathrm{n}=82) \\
(12 \%)\end{array}$ & $\begin{array}{l}\text { Did not receive } \\
\text { tocilizumab, } \\
(n=574)(88 \%)\end{array}$ & $p$ \\
\hline $\begin{array}{l}\text { Discharge, } \\
\mathrm{n}(\%)\end{array}$ & $\begin{array}{c}433 \\
(66.0)\end{array}$ & $\begin{array}{c}54 \\
(65.9)\end{array}$ & $\begin{array}{l}379 \\
(66.6)\end{array}$ & 0.975 \\
\hline $\begin{array}{l}\text { Death, } \\
\text { n (\%) }\end{array}$ & $\begin{array}{c}223 \\
(34.0)\end{array}$ & $\begin{array}{c}28 \\
(34.2)\end{array}$ & $\begin{array}{c}195 \\
(34.0)\end{array}$ & \\
\hline $\begin{array}{l}\text { ICU admission during follow-up, } n(\%) \\
(n=547)\end{array}$ & $\begin{array}{l}89 \\
(16)\end{array}$ & $\begin{array}{c}30 / 65 \\
(46)\end{array}$ & $\begin{array}{l}59 / 482 \\
(12)\end{array}$ & $<0.0001$ \\
\hline $\begin{array}{l}\text { Use of invasive mechanical } \\
\text { ventilation during follow-up, } n(\%) \\
(n=555)\end{array}$ & $\begin{array}{c}82 \\
(15)\end{array}$ & $\begin{array}{c}30 / 65 \\
(46)\end{array}$ & $\begin{array}{l}52 / 490 \\
(11)\end{array}$ & $<0.0011$ \\
\hline $\begin{array}{l}\text { Duration of mechanical ventilation } \\
\text { in survivors, days, median (IQR) } \\
(n=87)\end{array}$ & $\begin{array}{c}13 \\
(11-18)\end{array}$ & $\begin{array}{c}13 \\
(11-21) \\
n=27\end{array}$ & $\begin{array}{c}14 \\
(11-18) \\
n=60\end{array}$ & 0.9267 \\
\hline $\begin{array}{l}\text { Ventilator-free days in IMV survivors, } \\
\text { median (IQR) } \\
(n=87)\end{array}$ & $\begin{array}{c}12 \\
(7-17)\end{array}$ & $\begin{array}{c}10 \\
(6-16) \\
n=27\end{array}$ & $\begin{array}{c}12 \\
(8-17) \\
n=60\end{array}$ & 0.361 \\
\hline $\begin{array}{l}\text { Length of stay median in survivors, } \\
\text { days, median (IQR) } \\
(n=433)\end{array}$ & $\begin{array}{c}8 \\
(5-14)\end{array}$ & $\begin{array}{c}16 \\
(10-30) \\
n=54\end{array}$ & $\begin{array}{c}7 \\
(5-12) \\
n=379\end{array}$ & $<0.0001$ \\
\hline $\begin{array}{l}\text { Hospital-acquired infection, } \\
\mathrm{n}(\%)\end{array}$ & $\begin{array}{c}74 \\
(11.3)\end{array}$ & $\begin{array}{c}18 \\
(22.0)\end{array}$ & $\begin{array}{c}62 \\
(9.8)\end{array}$ & 0.001 \\
\hline $\begin{array}{l}\text { HAP/VAP, } \\
\text { n (\%) }\end{array}$ & $\begin{array}{l}49 \\
(7.5)\end{array}$ & $\begin{array}{c}13 \\
(15.9)\end{array}$ & $\begin{array}{c}36 \\
(6.3)\end{array}$ & 0.002 \\
\hline $\begin{array}{l}\text { Bloodstream infection, } \\
\mathrm{n}(\%)\end{array}$ & $\begin{array}{c}28 \\
(4.3)\end{array}$ & $\begin{array}{c}7 \\
(8.5)\end{array}$ & $\begin{array}{c}21 \\
(3.6)\end{array}$ & 0.041 \\
\hline $\begin{array}{l}\text { Hospital-acquired infection in } \\
\text { patients who received mechanical } \\
\text { ventilation, } \mathrm{n}(\%)\end{array}$ & $\begin{array}{l}72 / 183 \\
(39.3)\end{array}$ & $\begin{array}{l}18 / 47 \\
(38.3)\end{array}$ & $\begin{array}{l}54 / 136 \\
(39.7)\end{array}$ & 0.865 \\
\hline $\begin{array}{l}\text { Circulatory shock, } n(\%) \\
(n=653)\end{array}$ & $\begin{array}{c}169 \\
(25.9)\end{array}$ & $\begin{array}{c}41 \\
(50)\end{array}$ & $\begin{array}{c}128 \\
(22.5)\end{array}$ & $<0.0001$ \\
\hline $\begin{array}{l}\text { Renal replacement therapy, } n(\%) \\
(n=563)\end{array}$ & $\begin{array}{c}31 \\
(4.8)\end{array}$ & $\begin{array}{c}1 \\
(1.2)\end{array}$ & $\begin{array}{l}30 \\
(5.3)\end{array}$ & 0.161 \\
\hline
\end{tabular}

ICU: intensive care unit; IMV: invasive mechanical ventilation; IQR: interquartile range; HAP/VAP: hospital-acquired pneumonia/ventilatorassociated pneumonia.

laboratory tests, including $\mathrm{PaO}_{2} / \mathrm{FiO}_{2}$ ratio (116 vs. 116, $p=0.95$ ) (Table S4).

At 7 days after hospital admission, patients who received tocilizumab had lower CRP levels (3.0 vs. 10.5 $\mathrm{mg} / \mathrm{dL}, \mathrm{p}<0.01$ ) and higher median transaminase levels (72.9 vs. $41.6 \mathrm{U} / \mathrm{L}$ for ALT and 52.9 vs. $68 \mathrm{U} / \mathrm{L}$ for AST, $\mathrm{p}<0.01$ ). The median $\mathrm{PaO}_{2} / \mathrm{FiO}_{2}$ ratio was similar between groups ( 122 vs. $132, p=0.22$ ) (Table S5). No cases of fulminant hepatitis, intestinal perforation, or febrile neutropenia were observed.

\section{Outcomes}

Among 656/794 (85\%) patients with complete follow-up, 223/656 (34\%) died and 433/656 (66\%) were discharged. Among patients with incomplete follow-up, $124 / 138$ (90\%) were transferred to another facility and 14/138 (10\%) were discharged against medical advice for unspecified reasons (Fig. 1).

Outcomes are described in Table 2. No difference in hospital mortality was seen between groups (28/82 
[34\%] vs. 195/574 [34\%], $p=0.98$ ), even after excluding those who received the drug $\geq 24 \mathrm{~h}$ after IMV (12/52 [23\%] vs. 195/574 [34\%], p = 0.110). During follow-up, patients who received tocilizumab were more frequently admitted to the ICU (30/65 [46\%] vs. 59/482 [12\%], p < 0.01) and intubated (40/65 [46\%] vs. 52/490 [11\%], p < 0.01). IMV duration in survivors was similar between groups (13 vs. 13 days, $p=0.92$ ). No difference in VFDs at 28 days was observed between groups (median of 10 vs. 12 days, $p=0.36$ ). Circulatory shock was more common in the tocilizumab group (41/82 [50\%] vs. $128 / 574$ [22\%], $p<0.01$ ). The LOS in survivors was longer in the tocilizumab group (16 vs. 7 days, $p<0.01$ ). A total of 110 HAls were registered in 74 patients. Overall, HAls were more frequent in the tocilizumab group (18/82 [22\%] vs. $62 / 574$ [10\%], $p<0.01$ ). Hospital-acquired/ventilator-associated pneumonia (HAP/VAP) and bloodstream infection (BSI) were more frequent in the tocilizumab group, although no difference was seen after adjusting for mechanical ventilation use (18/47 [38\%] in the tocilizumab group vs. 54/136 [40\%] in the control group, $p=0.87$ ). The most frequent causes of HAP/VAP were Gram-negative bacilli, particularly Enterobacteriaceae, in $48 / 69$ isolates (70\%). The most frequent causes of BSI were coagulase-negative staphylococci in $14 / 35$ isolates ( $40 \%$ ). Invasive fungal infections included six episodes of candidemia and 14 episodes of COVID-19-associated aspergillosis (CAPA). No differences between groups were observed regarding candidemia ( 0 in the tocilizumab group vs. 6 in the non-tocilizumab group, $p=0.45$ ) or CAPA ( 3 in the tocilizumab group vs. 11 in the non-tocilizumab group, $\mathrm{p}=0.40$ ).

\section{Mortality}

Bivariate analysis showed increased hospital mortality with advanced age, male sex, DM, hypertension, higher Charlson score, higher Nutri-CoV category, lower $\mathrm{SpO}_{2}$ on admission, increased MuLBSTA and NEWS scores, lymphopenia, higher inflammatory marker levels, lower $\mathrm{PaO}_{2} / \mathrm{FiO}_{2}$ ratio at baseline, use of adjuvant steroids, and IMV. Tocilizumab was not associated with mortality (RR 1.0 [95\% Cl 0.73-1.39], $\mathrm{p}=0.98$ ). When analysis was restricted to patients who received IMV at any point, tocilizumab was not associated with decreased mortality (RR 0.76 [95\%Cl 0.531.10], $p=11$ ). In multivariate analysis, age, male sex, higher Nutri-CoV category, high CRP, and IMV, but not tocilizumab administration, were independently associated with increased mortality (Table 3 ). Tocilizumab administration before or within the first $24 \mathrm{~h}$ after IMV onset was not independently associated with decreased mortality in bivariable or multivariate analysis (RR 0.68 [95\%Cl 0.41-1.13], $\mathrm{p}=0.11$ ). A propensity score analysis using data from 586 patients with complete follow-up was estimated. The baseline variables included in the model were age, sex, DM, obesity, hypertension, chronic obstructive pulmonary disease, immunosuppression, health care worker status, $\mathrm{SpO}_{2} \leq 90 \%$, lymphopenia $<800$ cells $/ \mu \mathrm{L}$, CRP $>10 \mathrm{mg} / \mathrm{dL}, \mathrm{DD}>1000 \mathrm{ng} / \mathrm{mL}$, steroid use, and mechanical ventilation in the first $24 \mathrm{~h}$ after admission. The variance ratio of age (the only continuous variable included) was 1.21. Adequate balance within the matched sample was achieved (Table S6). A total of 73 treated patients were matched with 66 untreated controls. A regression adjustment for in-hospital mortality in the matched sample showed an adjusted odds ratio of $1.22(95 \% \mathrm{Cl} 0.52-2.86, \mathrm{p}=0.66)$ for tocilizumab treatment. No significant treatment effects were observed (difference 0.014, $p=872$ ) (Table 4).

\section{DISCUSSION}

We conducted a cohort study to describe the effect of tocilizumab in hospital mortality of patients with severe COVID-19. After a rigorous comparative analysis between groups, we observed that tocilizumab was not associated with a decrease in hospital mortality in patients with severe or critical COVID-19. Advanced age, male sex, DM, hypertension, increased disease severity, and selected laboratory tests results were associated with increased mortality in the bivariate analysis. After multivariate analysis, age, male sex, and the use of IMV were independently associated with increased mortality, as described in previous reports ${ }^{28-30}$. CRP $>10 \mathrm{mg} / \mathrm{dL}$ has been previously related to increased mortality, in agreement with our results $^{31}$. As previously reported ${ }^{26,32}$, increasing Nutri$\mathrm{CoV}$ category was also independently associated with mortality.

Numerous studies evaluating the efficacy of tocilizumab for COVID-19 have shown contradictory results. A retrospective cohort study by Biran et al. 
Table 3. Factors associated with mortality

\begin{tabular}{|c|c|c|}
\hline Characteristic & $\begin{array}{c}\mathrm{RR}(95 \mathrm{Cl} \%) \\
\mathrm{p}\end{array}$ & $\begin{array}{c}\text { aOR }(95 \% \mathrm{Cl}) \\
\mathrm{p}\end{array}$ \\
\hline Age & $\begin{aligned} 1.06 & (1.04-1.08) \\
& <0.001\end{aligned}$ & $\begin{aligned} 1.07 & (1.04-1.09), \\
& <0.001\end{aligned}$ \\
\hline Age $>60$ years $^{\mathrm{a}}$ & $\begin{array}{c}2.33(1.9-2.87) \\
<0.0001\end{array}$ & - \\
\hline Male sex & $\begin{array}{c}1.34(1.06-1.69) \\
0.0112\end{array}$ & $\begin{array}{c}1.78(1.12-2.83) \\
0.015\end{array}$ \\
\hline Diabetes mellitus ${ }^{a}$ & $\begin{array}{c}1.46(1.17-1.8) \\
0.0009\end{array}$ & - \\
\hline Hypertension & $\begin{array}{c}1.41(1.14-1.74) \\
0.002\end{array}$ & $\begin{array}{c}1.01(0.63-1.62) \\
0.964\end{array}$ \\
\hline Charlson comorbidity index $\geq 2^{b}$ & $\begin{aligned} 2.22 & (1.78-2.77) \\
& <0.0001\end{aligned}$ & - \\
\hline Nutri-CoV category & $\begin{aligned} 5.37 & (3.86-7.47) \\
& <0.001\end{aligned}$ & $\begin{aligned} 3.48 & (2.34-5.15) \\
& <0.001\end{aligned}$ \\
\hline Baseline $\mathrm{SpO}_{2} \leq 90 \%^{\mathrm{a}}$ & $\begin{array}{c}4.68(1.57-13.98) \\
0.0003\end{array}$ & - \\
\hline NEWS score $\geq 7^{b}$ & $\begin{aligned} 1.69 & (1.37-2.08) \\
& <0.0001\end{aligned}$ & - \\
\hline MuLBSTA score $\geq 11^{b}$ & $\begin{array}{c}2.00(1.64-2.45) \\
0.0001\end{array}$ & - \\
\hline $\begin{array}{l}\text { Absolute lymphocyte count } \\
<800 \text { cells } / \mu \mathrm{L}\end{array}$ & $\begin{aligned} 1.63 & (1.30-2.06) \\
& <0.0001\end{aligned}$ & $\begin{array}{c}1.41(0.90-2.21) \\
0.133\end{array}$ \\
\hline C-reactive protein $>10 \mathrm{mg} / \mathrm{dL}$ & $\begin{aligned} 3.78 & (2.51-5.67) \\
& <0.0001\end{aligned}$ & $\begin{array}{c}2.45(1.35-4.42) \\
0.003\end{array}$ \\
\hline Lactate dehydrogenase $>245 \mathrm{U} / \mathrm{L}^{\mathrm{c}}$ & $\begin{array}{c}8.36(2.75-25.41) \\
<0.0001\end{array}$ & - \\
\hline Troponin I $\geq 20 \mathrm{pg} / \mathrm{mL}^{\mathrm{c}}$ & $\begin{array}{c}3.00(2.48-3.63) \\
<0.0001\end{array}$ & - \\
\hline Ferritin $>500 \mathrm{ng} / \mathrm{mL}^{\mathrm{c}}$ & $\begin{array}{c}1.52(1.2-1.93) \\
0.0003\end{array}$ & - \\
\hline D-dimer $>1000 \mathrm{ng} / \mathrm{mL}$ & $\begin{array}{c}1.79(1.45-2.22) \\
<0.0001\end{array}$ & $\begin{array}{c}1.46(0.94-2.27) \\
0.094\end{array}$ \\
\hline $\mathrm{PaO}_{2} / \mathrm{FiO}_{2}$ ratio $<300^{c}$ & $\begin{array}{c}1.62(1.06-2.51) \\
0.016\end{array}$ & - \\
\hline Adjuvant steroids & $\begin{aligned} 1.77 & (1.39-2.26) \\
& <0.0001\end{aligned}$ & $\begin{array}{c}1.67(0.85-3.24) \\
0.130\end{array}$ \\
\hline ICU admission & $\begin{aligned} 2.1 & (1.71-2.57) \\
& <0.0001\end{aligned}$ & - \\
\hline Use of mechanical ventilation & $\begin{array}{c}1.95(1.6-2.39) \\
<0.0001\end{array}$ & $\begin{aligned} 3.01 & (1.78-5.10) \\
& <0.001\end{aligned}$ \\
\hline Treatment with tocilizumab & $\begin{array}{c}1.00(0.73-1.39) \\
0.9751\end{array}$ & $\begin{array}{c}1.09(0.58-2.06) \\
0.782\end{array}$ \\
\hline Hospital-acquired infection & $\begin{array}{c}1.29(0.98-1.71) \\
0.0865\end{array}$ & - \\
\hline
\end{tabular}

$\mathrm{N}=587$. PseudoR2 0.32 Area under the curve 0.86.

aAdvanced age, diabetes, and baseline $\mathrm{SpO}_{2}$ were not included in the model because they are included in the Nutri-CoV score.

${ }^{b}$ As Nutri-CoV score was included in the model, Charlson, NEWS, and MuLBSTA scores were not included.

'Elevated lactate dehydrogenase, troponin I, ferritin, and $\mathrm{PaO}_{2} / \mathrm{FiO}_{2}$ ratio were not included in the model to avoid excessive laboratory abnormalities that are known to be present in patients with severe COVID-19.

dICU admission was not included in the model because in our center, ICU admission is highly concordant with the use of mechanical ventilation. NEWS: National Early Warning Score; $\mathrm{FiO}_{2}$ : fraction of inspired oxygen; $\mathrm{mg} / \mathrm{dL}$ : milligram per deciliter; $\mathrm{ng} / \mathrm{mL}$ : nanogram per milliliter; PaO ${ }_{2}$ : partial pressure of oxygen in arterial blood; $\mathrm{pg} / \mathrm{mL}$ : picograms per milliliter; $\mathrm{U} / \mathrm{L}$ : units per liter; $\mu \mathrm{L}$ : microliter. 
Table 4. Treatment effect estimation

\begin{tabular}{lcccc}
\hline Sample & Treated & Untreated & $\begin{array}{c}\text { Coefficient } \\
(\mathrm{SE})\end{array}$ & $\begin{array}{c}95 \% \mathrm{Cl}, \\
\mathrm{p}\end{array}$ \\
\hline Treatment effect & 0.356 & 0.342 & 0.014 & $-0.153-0.180$, \\
& & & $(0.085)$ & 0.872 \\
\hline
\end{tabular}

SE: standard error.

reported lower mortality rates in critically ill patients who received tocilizumab (49\% vs. 61\%), including those on IMV10. Accordingly, two meta-analyses including data from cohort studies concluded that tocilizumab was associated with lower mortality $^{14,15}$. Another meta-analysis reported that tocilizumab decreased the probability of IMV ${ }^{16}$. Results from EMPACTA showed that tocilizumab reduced the likelihood of progression to mechanical ventilation or death ${ }^{17}$. Results from the RECOVERY study group reported lower mortality rates among patients who received tocilizumab ${ }^{18}$. In contrast, results from COVACTA revealed no difference in clinical status or 28-day mortality, but found a shorter median time to hospital discharge in patients who received tocilizumab $^{11}$. Similarly, another multicenter RCT by Stone et al. concluded that tocilizumab did not prevent intubation or death ${ }^{12}$. Recently, an open-label RCT carried out in India reported that tocilizumab did not reduce COVID-19 progression, in a health-care setting which may be more relatable to ours ${ }^{19}$. Furthermore, another meta-analysis using data from five RCT did not find reduced mortality ${ }^{13}$. In our study, patients received tocilizumab after a median of 11 days after symptom onset; so, it is likely that acute respiratory distress syndrome had been already established before the drug administration. We observed a non-significant trend for decreased mortality among patients who received tocilizumab before or within the first $24 \mathrm{~h}$ after IMV onset. Since 30\% received the drug more than $24 \mathrm{~h}$ after IMV onset, it is likely that late treatment had an impact on the outcome, although the optimal timing is yet to be proven ${ }^{33}$. A recently published observational study reported a lower 90-day mortality in patients that received tocilizumab within the first 10 days after symptom onset when compared with those that received the drug after day $11^{34}$. In our study, the prolonged time from admission to tocilizumab administration was due to low availability and high cost of the drug in our setting. Several differences were noted among patients who received tocilizumab due to the non-randomized nature of the study. Tocilizumab was more frequently used in males who may present with more severe forms of COVID-1935, and among health care workers, reflecting selection bias. Patients in the tocilizumab group had elevated CRP, $\mathrm{LDH}$, and ferritin levels during follow-up, which are known to be associated with increased mortality and may have led the decision to prescribe the drug ${ }^{36}$. Furthermore, patients who received tocilizumab were more likely to be admitted to the ICU for IMV and had a longer LOS, reflecting increased disease severity during follow-up. In an effort to minimize confounding, a propensity score matching analysis was performed, and no significant treatment effects were observed.

Tocilizumab was not associated with increased frequency of HAls after adjusting for IMV, which is compatible with previous RCT results ${ }^{11,12,17}$. Latent tuberculosis screening is not routinely recommended in rheumatological patients, but it has been a concern in countries with a high tuberculosis incidence during the pandemic ${ }^{37,38}$. Screening for latent tuberculosis was done in a low proportion of our patients. No cases of tuberculosis reactivation were diagnosed during follow-up. No other severe adverse events such as gastrointestinal perforation or febrile neutropenia related to tocilizumab were recorded.

During the study period, steroid treatment was not yet part of the standard of care, and those who received them were frequently diagnosed with refractory circulatory shock, explaining the increased mortality seen on bivariate analysis for this subset of patients. Of note, the fact that tocilizumab, in contrast with the EMPACTA and RECOVERY trials ${ }^{17,18}$, was not administered with steroids, could have played a role in our results. The COVACTA and BACC Bay trials, which did not find differences in mortality, also reported a lower corticosteroid use in the tocilizumab group $^{11,12}$. 
Baseline demographic characteristics were consistent with previous reports from our country, which include a high prevalence of obesity, DM, and hypertension in COVID-19 patients and in the general population ${ }^{39,40}$. Alarmingly, a high use of unnecessary antibiotics was found ${ }^{41-44}$.

Our study has several limitations. This was an observational cohort study with unbalanced groups even after adjusting for confounders. It is possible that a small sample size rendered insufficient power to detect differences in mortality similar to those reported in clinical trials ${ }^{11,12,17,18}$. Tocilizumab use was not systematic regarding dose and timing, which led to variability of prescriptions, although a response in laboratory results in the first $48 \mathrm{~h}$ was seen, as previously described ${ }^{45}$. Furthermore, $17 \%$ of patients were lost to follow-up and not included in outcome analysis. The main reasons for this were improvement and transfer to a convalescence center or transfer to other hospitals with available ICU space at the time due to disease progression. ICU unavailability during the peak of the pandemic contributes to mortality ${ }^{46}$.

We consider that this study reflects non-systematic and individual physician-based prescription during the early months of the pandemic. This analysis also allows a description of adverse events, including HAls. The late use of tocilizumab or other immunomodulatory treatments is a real-world situation in countries where drugs are costly and not widely available. We believe that this was a common practice during the study period since high-impact trials were not yet published. This report provides additional evidence about the effect and safety of tocilizumab for COVID-19 in settings outside controlled clinical trials, where the health-care system is constantly overrun, and some treatments may not be widely available.

In conclusion, we did not observe an association between tocilizumab and reduced hospital mortality among patients with severe or critical COVID-19. Advanced age, male sex, elevated inflammatory markers, and mechanical ventilation were associated with increased mortality. Tocilizumab appeared to be safe, as no severe adverse effects were registered. Even though no benefit on mortality was observed, early administration may be beneficial. Physicians should consider setting-specific factors when prescribing tocilizumab.
In Mexico, drug availability and cost could represent barriers for optimal drug timing and dosing. The present study, underscores that tocilizumab, if considered for therapy, should be used in strict adherence to current guidelines and high-quality evolving evidence.

\section{SUPPLEMENTARY DATA}

Supplementary data are available at DOI: $10.24875 /$ RIC.21000404. These data are provided by the corresponding author and published online for the benefit of the reader. The contents of supplementary data are the sole responsibility of the authors.

\section{REFERENCES}

1. Wu C, Chen X, Cai Y, Xia J, Zhou X, Xu S, et al. Risk factors associated with acute respiratory distress syndrome and death in patients with coronavirus disease 2019 pneumonia in Wuhan, China. JAMA Intern Med. 2020;180:934-43.

2. Del Valle DM, Kim-Schulze S, Huang HH, Beckmann ND, Nirenberg $\mathrm{S}$, Wang $\mathrm{B}$, et al. An inflammatory cytokine signature predicts COVID-19 severity and survival. Nat Med. 2020; 26:1636-43.

3. RECOVERY Collaborative Group. Dexamethasone in hospitalized patients with Covid-19. N Engl J Med. 2020;384:693-704.

4. Zhang Y, Chen Y, Meng Z. Immunomodulation for severe COVID-19 pneumonia: the state of the art. Front Immunol. 2020; 11:577442.

5. Kang S, Tanaka T, Narazaki M, Kishimoto T. Targeting interleukin-6 signaling in clinic. Immunity. 2019;50:1007-23.

6. Singh JA, Saag KG, Bridges SL, AkI EA, Bannuru RR, Sullivan MC, et al. 2015 American college of rheumatology guideline for the treatment of rheumatoid arthritis. Arthritis Care Res (Hoboken). 2016;68:1-25.

7. Shimabukuro-Vornhagen A, Gödel P, Subklewe M, Stemmler HJ, Schlößer HA, Schlaak M, et al. Cytokine release syndrome. J Immunother Cancer. 2018;6:56

8. Xu X, Han M, Li T, Sun W, Wang D, Fu B, et al. Effective treatment of severe COVID-19 patients with tocilizumab. Proc Nat Acad Sci U S A. 2020;117:10970-5.

9. Toniati P, Piva S, Cattalini M, Garrafa E, Regola F, Castelli F, et al. Tocilizumab for the treatment of severe COVID-19 pneumonia with hyperinflammatory syndrome and acute respiratory failure: a single center study of 100 patients in Brescia, Italy. Autoimmun Rev. 2020;19:102568.

10. Biran N, Ip A, Ahn J, Go RC, Wang S, Mathura S, et al. Tocilizumab among patients with COVID-19 in the intensive care unit: a multicentre observational study. Lancet Rheumatol. 2020; 2:e603-12.

11. Rosas IO, Bräu N, Waters M, Go RC, Hunter BD, Bhagani S, et al. Tocilizumab in hospitalized patients with severe Covid-19 pneumonia. N Engl J Med. 2021;384:1503-16.

12. Stone JH, Frigault MJ, Serling-Boyd NJ, Fernandes AD, Harvey L, Foulkes AS, et al. Efficacy of tocilizumab in patients hospitalized with Covid-19. N Engl J Med. 2020;383:2333-44.

13. Huang YT, Chao CM, Lai CC. Impact of tocilizumab on the mortality of patients with coronavirus disease 2019. Clin Infect Dis. 2020;72:e1156-7.

14. Berardicurti O, Ruscitti P, Ursini F, D’Andrea S, Ciaffi J, Meliconi R, et al. Mortality in tocilizumab-treated patients with COVID-19: a systematic review and meta-analysis. Clin Exp Rheumatol. 2020;38:1247-54.

15. Malgie J, Schoones JW, Pijls BG. Decreased mortality in coronavirus disease 2019 patients treated with tocilizumab: a rapid 
systematic review and meta-analysis of observational studies. Clin Infect Dis. 2020;72:e742-9.

16. Tleyjeh IM, Kashour Z, Damlaj M, Riaz M, Tlayjeh H, Altannir M, et al. Efficacy and safety of tocilizumab in COVID-19 patients: a living systematic review and meta-analysis. Clin Microbiol Infect. 2021;27:215-27.

17. Salama C, Han J, Yau L, Reiss WG, Kramer B, Neidhart JD, et al. Tocilizumab in patients hospitalized with Covid-19 pneumonia. N Engl J Med. 2021;384:20-30.

18. Abani O, Abbas A, Abbas F, Abbas M, Abbasi S, Abbass $\mathrm{H}$, et al. Tocilizumab in patients admitted to hospital with COVID-19 (RECOVERY): a randomised, controlled, open-label, platform trial RECOVERY collaborative group. Lancet. 2021;397:1637-45.

19. Soin AS, Kumar K, Choudhary NS, Sharma P, Mehta Y, Kataria S, et al. Tocilizumab plus standard care versus standard care in patients in India with moderate to severe COVID-19-associated cytokine release syndrome (COVINTOC): an open-label, multicentre, randomised, controlled, phase 3 trial. Lancet Respir Med. 2021;9:511-21.

20. Bhimraj A, Morgan RL, Shumaker AH, Lavergne V, Baden L, Cheng VC, et al. Infectious diseases society of America guidelines on the treatment and management of patients with $\mathrm{CO}$ VID-19. Clin Infect Dis. 2021;4:1-20.

21. World Health Organizaion. Clinical Management of COVID-19: interim Guidance. Geneva: World Health Organization; 2021. Available from: https://www.apps.who.int/iris/handle/ 10665/338882. [Last accessed on 2021 Aug 23].

22. Alhazzani W, Evans L, Alshamsi F, Møller MH, Ostermann M, Prescott HC, et al. Surviving sepsis Campaign guidelines on the management of adults with coronavirus disease 2019 (COVID-19) in the ICU: first update. Crit Care Med. 2021;49:e219-34.

23. COVID-19 Treatment Guidelines Panel. Coronavirus Disease 2019 (COVID-19) Treatment Guidelines. National Institutes of Health. Available from: https://www.covid19treatmentguidelines.nih.gov. [Last accessed on 2021 Aug 23].

24. Wu Z, McGoogan JM. Characteristics of and important lessons from the coronavirus disease 2019 (COVID-19) outbreak in China: summary of a report of 72314 cases from the Chinese center for disease control and prevention. J Am Med Assoc. 2020;323:1239-42.

25. Bello-Chavolla OY, Bahena-López JP, Antonio-Villa NE, VargasVázquez A, González-Díaz A, Márquez-Salinas A, et al. Predicting mortality due to SARS-CoV-2: a mechanistic score relating obesity and diabetes to COVID-19 outcomes in Mexico. J Clin Endocrinol Metab. 2020;105;dgaa346.

26. Bello-Chavolla OY, Antonio-Villa NE, Ortiz-Brizuela E, VargasVázquez A, González-Lara MF, De Leon AP, et al. Validation and repurposing of the MSLCOVID-19 score for prediction of severe COVID-19 using simple clinical predictors in a triage setting: the nutri-CoV score. PLoS One. 2020;15:e0244051.

27. Corman VM, Landt O, Kaiser M, Molenkamp R, Meijer A, Chu DK, et al. Detection of 2019-nCoV by RT-PCR. Euro Surveill. 2020;25:1-8.

28. Rosenthal N, Cao Z, Gundrum J, Sianis J, Safo S. Risk factors associated with in-hospital mortality in a US national sample of patients with COVID-19. JAMA Netw Open. 2020;3:e2029058.

29. Williamson EJ, Walker AJ, Bhaskaran K, Bacon S, Bates C, Morton CE, et al. Factors associated with COVID-19-related death using OpenSAFELY. Nature. 2020;584:430-6.

30. Li X, Xu S, Yu M, Wang K, Tao Y, Zhou Y, et al. Risk factors for severity and mortality in adult COVID-19 inpatients in Wuhan. J Allergy Clin Immunol. 2020;146:110-8.

31. Chidambaram V, Tun NL, Haque WZ, Gilbert Majella M, Kumar Sivakumar R, Kumar A, et al. Factors associated with disease severity and mortality among patients with COVID-19: a systematic review and meta-analysis. PLoS One. 2020;15:1-29

32. Soto-Mota A, Marfil-Garza BA, de Obeso SC, Martínez E, Carrillo-Vázquez DA, Tadeo-Espinoza $\mathrm{H}$, et al. Prospective predictive performance comparison between clinical gestalt and validated COVID-19 mortality scores. MedRxiv. 2021;???:???.

33. Rizk JG, Kalantar-Zadeh K, Mehra MR, Lavie CJ, Rizk Y, Forthal DN. Pharmaco-immunomodulatory therapy in COVID-19. Drugs. 2020;80:1267-92

34. Moreno Díaz R, Amor García MA, Teigell Muñoz FJ, Saldaña Perez LE, Mateos González M, Melero Bermejo JA, et al. Does timing matter on tocilizumab administration? Clinical, analytical and radiological outcomes in COVID-19. Eur J Hosp Pharm. 2021;2021:1-6.

35. Pradhan A, Olsson PE. Sex differences in severity and mortality from COVID-19: are males more vulnerable? Biol Sex Differ 2020;11:1-11

36. Zhou F, Yu T, Du R, Fan G, Liu Y, Liu Z, et al. Clinical course and risk factors for mortality of adult inpatients with COVID-19 in Wuhan, China : a retrospective cohort study. Lancet. 2020; 395:1054-62.

37. Woodman M, Haeusler IL, Grandjean L. Tuberculosis genetic epidemiology: a Latin American perspective. Genes (Basel). 2019;10:53.

38. Lin CT, Huang WN, Hsieh CW, Chen YM, Chen DY, Hsieh TY, et al. Safety and effectiveness of tocilizumab in treating patients with rheumatoid arthritis-a three-year study in Taiwan. J Microbiol Immunol Infect. 2019;52:141-50.

39. Ortiz-Brizuela E, Villanueva-Reza M, González-Lara MF, TamezTorres KM, Román-Montes CM, Díaz-Mejía BA, et al. Clinical and epidemiological characteristics of patients diagnosed with Covid-19 in a tertiary-care center in Mexico city: a prospective cohort study. Rev Invest Clin. 2020;72:165-77.

40. Alcalde-Rabanal JE, Orozco-Núñez E, Espinosa-Henao OE, Arredondo-López A, Alcayde-Barranco L. The complex scenario of obesity, diabetes and hypertension in the area of influence of primary healthcare facilities in Mexico. PLoS One. 2018; 13:1-17.

41. Abelenda-Alonso G, Padullés A, Rombauts A, Gudiol C, Pujol M, Alvarez-Pouso $C$, et al. Antibiotic prescription during the COVID-19 pandemic: a biphasic pattern. Infect Control Hosp Epidemiol. 2020;41:1371-2

42. Lansbury L, Lim B, Baskaran V, Lim WS. Co-infections in people with COVID-19: a systematic review and meta-analysis. J Infect. 2020;81:266-75.

43. Rawson TM, Moore LS, Castro-Sánchez E, Charani E, Davies F, Satta G, et al. COVID-19 and the potential long-term impact on antimicrobial resistance. J Antimicrob Chemother. 2020;75: 1681-4.

44. Martínez-Guerra BA, González-Lara MF, De-León-Cividanes NA, Tamez-Torres KM, Román-Montes CM, Rajme-López S, et al. Antimicrobial resistance patterns and antibiotic use during hospital conversion in the COVID-19 pandemic. Antibiotics. 2021; 10:182.

45. Capra R, De Rossi N, Mattioli F, Romanelli G, Scarpazza C, Sormani MP, et al. Impact of low-dose tocilizumab on mortality rate in patients with COVID-19 related pneumonia. Eur J Intern Med. 2020;76:31-5

46. Olivas-Martínez A, Cárdenas-Fragoso JL, Jiménez JV, LozanoCruz OA, Ortiz-Brizuela E, Tovar-Méndez VH, et al. In-hospital mortality from severe COVID-19 in a tertiary care center in Mexico city; causes of death, risk factors and the impact of hospital saturation. PLoS One. 2021;16:e0245772. 